Enrico Pogliani

Enrico Clini

\section{Association therapy as a prognostic factor in deep fungal infection complicating oncohaematological diseases}

E. Pogliani, M. D.

Internal Medicine Department,

Haematology Division,

University of Milan,

S. Gerardo Hospital,

Monza (Milan), Italy

E. Clini, M. D. (两)

Cardiopulmonary Division,

Clinica del Lavoro Foundation IRCSS,

Medical Centre of Rehabilitation,

Via Pinidolo, 23,

I-25064 Gussago (BS), Italy

Tel.: 030-25281

Fax: 030-2521718

\begin{abstract}
A group of 31 oncohaemopathic patients (17 male, mean age $44 \pm 6$ years), diagnosed as having primary deep fungal infection involving the lungs, were retrospectively evaluated. When infection was suspected on a clinical basis the major associated risks for death were the duration of bone marrow aplasia $(12 \pm 7$ versus $21 \pm 6$ days, $P<0.001$ ), increase in white blood cells and, in particular, prolonged granulocytopenia $(11 \pm 5$ versus $24 \pm 8$ days, $P<0.001$ ) when survivors were compared with patients, who died. Our therapeutic empirical approach was based on the association of i.v. amphotericin $\mathrm{B}, 1 \mathrm{mg} \mathrm{kg}^{-1} \mathrm{day}^{-1}$, with oral 5fluorocytosine (5-FC) $150 \mathrm{mg} \mathrm{kg}^{-1}$ day $^{-1}$. Only 9 subjects received combination therapy for more than 7 days. For majority of them, oral 5-FC was interrupted because of
\end{abstract}

altered compliance or sustained liver damage. A $\chi^{2}$ test for independent parameters showed $(P=0.0021)$ a concentration of deaths among patients who received amphotericin B alone (15/ $22)$; none of the patients treated with amphotericin B +5-FC ( 9 cases) died. Results generally suggest that a more favourable outcome was statistically associated with empirical antifungal combination therapy in deep fungal infection, although both treatment regimens showed effectiveness in terms of survival. Nevertheless the low 5-FC compliance and the small sample do not indicate the safe use of this drug in a large population.

Key words Deep fungal infection Pulmonary aspergillosis - Antifungal therapy - Granulocytopenia Haematological malignancies

\section{Introduction}

Fungal infections are commonly associated with death among leukaemic patients undergoing chemotherapy $[3,5]$. Pulmonary infection due to invasive aspergillosis appears as one of the most frequent causes of morbidity in neutropenic (polymorphonuclear neutrophils $<500 / \mu \mathrm{l})$ leukaemic patients $[2,11,13]$ often associated with a high mortality rate [19]. Difficulties deriving from in vivo diagnostic procedures partly explain the poor outcome of these infections $[1,16]$.
Risk factors for developing deep fungal infection (DFI) have been extensively studied, and granulocytopenia [9], steroids or a prolonged broad-spectrum antibiotic regimen $[16,19]$ and haematological remission [17] appear to be the most frequently associated causes.

Empirical pharmacological antifungal treatment is still the most common approach when DFI is suspected on a clinical basis. In this report we retrospectively evaluated the role of antifungal therapy in relationship. to the outcome of DFI in a group of oncohaemopathic patients. 


\section{Patients and methods}

A group of 31 consecutive oncohaemopathic patients (17 male), diagnosed for suspected DFI with primary pulmonary involvement and treated with early empirical antifungal therapy, were evaluated. In Table 1 patient characteristics are summarized. All the subjects presented suspected DFI during aplasia due to the haematological illness or the drugs employed. Patients were experiencing a febrile episode (temperature $>38^{\circ} \mathrm{C}$ ), and a treatment of broad-spectrum antibiotics (amikacin plus ceftazidime plus vancomycin) was instituted according to the most recent advice [6]. In all cases lung involvement was proven after X-ray examination.

Twice a day blood cultures were obtained and twice a week samples from stools, urine, nasal and bronchial secretions (aspirates and/or broncho-alveolar lavage, BAL) were taken to obtain diagnostic confirmation. Microbiological research was conducted on BAL samples to exclude infections from other pathogens (Pneumocystis carinii, Legionella, viruses and rare bacterial species).

Fever refractory to antibacterial therapy for more than 5 days was then treated with empirical antifungal therapy based upon the association between i.v. amphotericin B (AmpB) at a mean dose of $1 \mathrm{mg} \mathrm{kg}^{-1} \mathrm{day}^{-1}$ and oral 5-fluorocytosine (5-FC) at a dose of $150 \mathrm{mg} \mathrm{kg}^{-1} \mathrm{day}^{-1}$. A close monitoring of the side-effects of these drugs was performed. Early liver damage due to the use of 5 -FC determined drug suspension within 7 days in 22 patients; two therapy groups were therefore selected. The mean time of antifungal treatment was $27 \pm 4$ days and this did not significantly differ between the therapy groups. An oral nistatin regimen (three times daily) was instituted as an anti-Candida prophylactic therapy. Onset of DFI, defined according the criteria of Gerson $[8,10]$, age, duration of granulocytopenia, aplasia, leukaemic status, white blood cell (WBC) count and the kind, dose and duration of antifungal therapies were correlated to the vital prognosis.

Student's $t$-test was applied for the variables considered in the two groups, according to the outcome of the infective episode. Moreover a $\chi^{2}$ test was performed in the therapy groups, always related to vital prognosis. A $P$ value less than 0.05 was considered statistically significant.

\section{Results}

The mortality rate for DFI complicating haematological malignancies was approximately $50 \%$ in our sample (16 out of 31 patients).

Culture from BAL was possible in 9 out of 31 cases. Microbiological confirmation of DFI was obtained in 24 cases $(84 \%)$; in 2 out of 9 patients a positive BAL culture was obtained. Aspergillus species were present in $90 \%$ of positive cases ( 22 out of 24 corresponding to $2 / 3$ of the total sample) and Cryptococcus was identified in 5 out of 24 patients (20\%). Cryptococcus was isolated from BAL and serological confirmation was obtained at the same time. For 7 patients $(16 \%)$ there was no microbiological confirmation for their suspected DFI; 2 of them completed an antifungal regimen with both AmpB and 5-FC. The mean time of onset of DFI did not differ significantly between survivors and nonsurvivors ( $15 \pm 5$ and $17 \pm 3$ days respectively) but the
Table 1 Patients' characteristics. ( $A M L$ Acute myelocytic leukaemia, $A L L$ acute lymphocytic leukaemia, $C M L-B C$ blastic crisis of chronic myelogenous leukaemia)

\begin{tabular}{lc}
\hline Age (years) & $44 \pm 6$ \\
Range & $16-52$ \\
Gender (M/F) & $17 / 14$ \\
Malignancy & \\
AML & 15 \\
ALL & 11 \\
CML-BC & 2 \\
Lymphoma & 3 \\
Complete remission & 20 \\
Residual leukaemia & 11 \\
Microbiological confirmation & 24 \\
\hline
\end{tabular}

Table 2 Risk factor analysis associated with the outcome of deep fungal infection $(D F I)$. (AmpB Amphotericin B, 5-FC 5-fluorocytosine, $W B C$ white blood cells)

\begin{tabular}{lcll}
\hline Potential risk factor & $\begin{array}{l}\text { Survivors } \\
(n=16)\end{array}$ & $\begin{array}{l}\text { Non-survivors } \\
(n=15)\end{array}$ & $P$ \\
\hline Age (years) & $43 \pm 5$ & $45 \pm 2$ & NS \\
AML & $7 / 16$ & $8 / 15$ & NS \\
ALL & $6 / 16$ & $5 / 15$ & NS \\
BC & $1 / 16$ & $1 / 15$ & NS \\
Lymphoma & $2 / 16$ & $1 / 15$ & NS \\
Aplasia (days) & $12 \pm 7$ & $21 \pm 6$ & $<0.001$ \\
Onset of DFI (days) & $15 \pm 5$ & $17 \pm 3$ & NS \\
Granulocytopenia (days) & $11 \pm 5$ & $24 \pm 8$ & $<0.001$ \\
Treatment AmpB & $7 / 22$ & $15 / 22$ & $<0.01$ \\
Treatment AmpB +5-FC & $9 / 9$ & $0 / 9$ & $<0.0001$ \\
Remission & $12 / 16$ & $8 / 15$ & $\mathrm{NS}$ \\
Increased WBC count & $12 / 16$ & $2 / 15$ & $<0.0001$ \\
\hline
\end{tabular}

duration of bone marrow aplasia and granulocytopenia were strictly related to the prognosis $(P<0.001$ between the two groups) as well the increase in white blood cells $(P<0.0001)$.

We observed a significant difference $(P<0.05)$ between groups when the white blood cell increase was compared according to the different treatments adopted; the number of patients who presented a cell increase was higher in the $\mathrm{AmpB}+5$-FC group. Moreover the white cell count at the onset of a febrile episode and evidence of pulmonary infiltration were similar in both groups.

Haematological remission was present in 12 out of 16 survivors and in 8 out of 15 of those who died, but the difference was not significant. No differences in age or kind of malignancy were found, as illustrated in Table 2 .

If we look at the treatment groups $(\mathrm{AmpB}+5-\mathrm{FC}$ or $\mathrm{AmpB}$ ), 15 out of 22 patients died in the AmpB group while 0 out of 9 died in the AmpB +5-FC group. There were 7 and 9 survivors respectively in the two therapy groups. $\chi^{2}$ analysis for independent parameters, applied to these groups according to the outcome, re- 
vealed a significant concentration of deaths in the AmpB group $(P=0.0021)$. No differences were observed among the survivors in terms of the duration of different treatments (26 \pm 9 days for $\mathrm{AmpB}$ and $24 \pm 5$ days for $\mathrm{AmpB}+5-\mathrm{FC})$.

\section{Discussion}

The present results principally emphasize the better outcome for DFI retrospectively encountered in the group of oncohaemopathic patients treated with antifungal combination therapy $(\mathrm{AmpB}+5-\mathrm{FC})$.

The use of systemic AmpB is still relevant for the treatment of DFI in leukaemic patients especially when an empirical approach cannot be delayed [12]. A prophylactic use of AmpB is also proposed in patients who have been previously diagnosed as having DFI [18]. Pharmacological studies $[4,15]$ suggest the rationale for the use of an associated antifungal therapy that can provide a wide spectrum and possibly a synergistic effect, as requested in our sample.

Although combination therapy was considered to offer no advantages in terms of survival [20], our results seem to encourage the use of associated 5-FC. Nevertheless 5-FC compliance and hepatic failure (the causes for suspension of 5-FC) could be related to a more severe fungal infection and a poorer prognosis. In fact, AmpB also significantly improved the vital prognosis for the infection (see Table 2).

Other results on the risk factors associated with a poor outcome for this infection generally reflect, in our study, the recent suggestions of Ribrag [17] except for the remission of the underlying disease, which was higher in terms of the number of survivors but not significantly linked with a better outcome. Microbiological confirmation for aspergillosis was higher than that quoted in the literature [7] but we cannot exclude the possibility of contamination from nasal secretion (positive for Aspergillus species in 29/31 cases and in 22/22 patients with positive bronchial secretion culture) as an adjunctive risk for pulmonary aspergillosis during a neutropenic phase [14]. No nasal antifungal prophylaxis was adopted. A presumptive diagnosis of pulmonary aspergillosis (or at least fungal infection) in the nonmicrobiologically confirmed patients was made according to the criteria of Gerson $[8,10]$ and after infection from other pathogens had been excluded (see Patients and methods).

In conclusion we retrospectively found that the use of a combination antifungal therapy could be effective in suspected DFI complicating haematological malignancies. The low 5-FC compliance does not warrant the empirical use of this drug in a large population.

\section{References}

1. Albelda S, Talbot GH, Gerson SI, et al (1984) The role of fiberoptic bronchoscopy in the diagnosis of invasive pulmonary aspergillosis in patients with acute leukemia. Am J Med 76:1027--1034

2. Bodey $\mathrm{G}$ (1986) Fungal infection and fever of unknown origin in neutropenic patients. Am J Med 80 [Suppl 5Cl:112-119

3. Chang $\mathrm{H}$, Rodriguez V, Narboni G, Bodey G, Luna M, Freirech E (1976) Causes of death in adults with acute leukemia. Medicine 55:259-264

4. Craven P, Graybill JR (1984) Combination of oral flucytosine and ketoconazole as therapy for experimental cryptococcal meningitis. J Infect Dis 149:584-589

5. De Gregorio M, Lee W, Linker C, Jacobs R, Ries C (1982) Fungal infections in patients with acute leukemia. Am J Med 73:543-548
6. EORTC and National Cancer Institute of Canada (1992) Vancomycin added to empirical combination antibiotic therapy for fever in granulocytopenic cancer patients. J Infect Dis 163:951-958

7. Fischer BD, Armstrong D, et al (1981) Invasive aspergillosis progress in early diagnosis and treatment. Am J Med 71:571-577

8. Gerson SL, Talbot GH (1985) Discriminant scorecard for diagnosis of invasive pulmonary aspergillosis in patients with acute leukemia. Am J Med 79:57-64

9. Gerson SL, Talbot GH, GH, Hurwitz $S$, et al (1984) Prolonged granulocytopenia: the major risk factor for invasive pulmonary aspergillosis in patients with acute leukemia. Intern Med 100:345-351

10. Gerson SL, Talbot GH, Luke S, et al (1985) Invasive pulmonary aspergillosis in adult acute leukemia: clinical clues to its diagnosis. J Clin Oncol 3:1109-1116
11. Hebert PA, Bayer AS (1981) Fungal pneumonia. Invasive pulmonary aspergillosis. Chest 80:220-225

12. Holleran W, Wilbur J, De Gregorio M (1985) Empiric amphotericin B therapy in patients with acute leukemia. Am J Med 7:619-624

13. Krick J, Remington J (1976) Opportunistic invasive fungal infections in patients with leukaemia and lymphoma. Clin Haematol 5:249--254

14. Martino P, Raccah R, Gentile G, et al (1989) Aspergillus colonization of the nose and pulmonary aspergillosis in neutropenic patients: a retrospective study. Haematologica 74:263-265

15. Medoff $G$ (1983) Antifungal agents useful in the therapy of systemic fungal infection. Am Rev Pharmacol Toxicol 23:303-309 
16. Nalesnik M, Myerowitz R, Jenkins R et al (1980) Significance of Aspergillus species isolated from respiratory secretions in the diagnosis of invasive pulmonary aspergillosis. I Clin Microbiol 11:370-376

17. Ribrag V, Dreyfus F, Venot A, Leblong V, Lanore JJ, Varet B (1993) Prognostic factors of invasive pulmonary aspergillosis in leukemic patients. Leukemia Lymphoma 10:317321
18. Robertson M, Larson R (1988) Recurrent fungal pneumonias in patients with acute non-lymphocytic leukemia undergoing multiple courses of intensive chemotherapy. Am J Med $84: 233-239$
19. Schwartz RS, Mackintosh F, Schrier S, Greenberg P (1984) Multivariate analysis of factors associated with invasive fungal disease during remission induction therapy for acute leukemia. Cancer 53:411-419

20. Zuger A, Louie E, Holzman RS, Simberkoff MS, Rahal JJ (1986) Cryptococcal disease in patients with AIDS: diagnostic features and outcome of treatment. Ann Intern Med 104:234 238 